\title{
Formation of solitons in a waveguide at second harmonic generation
}

\author{
Sergey Sazonov, Alexey Kalinovich*, Irina Zakharova, Mariya Komissarova, and \\ Pavel Shestakov \\ Lomonosov Moscow State University, Faculty of Physics, Leninskie Gory, Moscow, 119991 Russia
}

\begin{abstract}
Second harmonic generation in a quadratic nonlinear waveguide is analyzed analytically and numerically. Formation of spatio-temporal solitons is demonstrated. They can form both positive and negative coefficients of group velocity dispersion due to the waveguide geometry. Analytical results are confirmed by computer simulations.
\end{abstract}

The propagation of a pulse-beam of optical radiation in a planar waveguide with cubic nonlinearity is accompanied by a space-time collapse, the nature of which depends on the input parameters. A medium with quadratic nonlinearity has long been used to avoid collapse [1] and to generate a stable spatio-temporal solitons.

In most theoretical works devoted to spatio-temporal solitons in quadratic nonlinearity, studies were conducted for a uniform medium [1-4]. In a waveguide of the same geometry in addition to the competition between nonlinearity, dispersion and diffraction, focusing properties of the waveguide play an important role [3, 5].

Pulsed second harmonic generation in a planar waveguide with a parabolic profile of the refractive index at the combined effect of diffraction and dispersion is described by the following system of equations for the complex amplitudes of the fundamental frequency $\psi_{1}$ and second harmonic $\psi_{2}$ :

$$
\begin{aligned}
& i\left[\frac{\partial \psi_{1}}{\partial z}+\left(\varepsilon_{1} \alpha_{1} \frac{x^{2}}{a_{1}^{2}}+\delta\right) \frac{\partial \psi_{1}}{\partial \tau}\right]+\frac{\beta_{2}^{(1)}}{2} \frac{\partial^{2} \psi_{1}}{\partial \tau^{2}}-\gamma_{1} \psi_{1}^{*} \psi_{2} e^{i\left(2 k_{1}-k_{2}\right) z}=\varepsilon_{1} q_{1} \frac{x^{2}}{a_{1}^{2}} \psi_{1}+\frac{c}{2 n_{1} \omega} \frac{\partial^{2} \psi_{1}}{\partial x^{2}} \\
& i\left[\frac{\partial \psi_{2}}{\partial z}+\left(\varepsilon_{2} \alpha_{2} \frac{x^{2}}{a_{2}^{2}}-\delta\right) \frac{\partial \psi_{2}}{\partial \tau}\right]+\frac{\beta_{2}^{(2)}}{2} \frac{\partial^{2} \psi_{2}}{\partial \tau^{2}}-\gamma_{2} \psi_{1}^{2} e^{-i\left(2 k_{1}-k_{2}\right) z}=2 \varepsilon_{2} q_{2} \frac{x^{2}}{a_{2}^{2}} \psi_{2}+\frac{c}{4 n_{2} \omega} \frac{\partial^{2} \psi_{2}}{\partial x^{2}} .
\end{aligned}
$$

Here, $n_{1,2}$ are the refractive indices for the first and second harmonics in the center of the waveguide, $\beta_{2}^{(1,2)}$ are the coefficients of group velocity dispersion, $\tau=t-\frac{1}{2}\left(\frac{1}{v_{g 1}}+\frac{1}{v_{g 2}}\right) z$, $\gamma_{1}=\frac{4 \pi \omega}{c n} \chi_{2}(-\omega ; 2 \omega), \quad \gamma_{2}=\frac{4 \pi \omega}{c n} \chi_{2}(\omega ; \omega), \delta=\frac{1}{2}\left(\frac{1}{v_{g 1}}-\frac{1}{v_{g 2}}\right), \alpha_{1,2}=\frac{n_{1,2}^{2}-1}{2 c n_{1,2}}, q_{1,2}=\omega \alpha_{1,2}$.

" Corresponding author: kalinovich@gmail.com 
Possible modes of propagation of the pulse beams at the main and doubled frequencies depend on the competition between the focusing properties of nonlinearity and the focusing properties of the waveguide, as well as from the critical parameter, $\eta=0,43 \frac{n^{2}}{n^{2}-1} \frac{a^{2} l_{D}}{l_{d i s}^{3}}$, where $l_{d i s}=\frac{2 \tau_{0}^{2}}{\left|\beta_{2}\right|}$ - the dispersion length, $l_{D}=\frac{n \omega}{c} R_{0}^{2}-$ the diffraction length. There are four main scenarios:

$1)$ under defocusing nonlinearity $\left(\beta_{2}>0\right)$ and defocusing waveguide $\left(\varepsilon_{1,2}=+1\right)$ there are no stable modes of propagation;

$2)$ under defocusing nonlinearity $\left(\beta_{2}>0\right)$ and a focusing waveguide $\left(\varepsilon_{1,2}=-1\right)$ the formation of solitons due to waveguide geometry is possible;

3 ) at focusing nonlinearity $\left(\beta_{2}<0\right)$ and defocusing waveguide $\left(\varepsilon_{1,2}=+1\right)$ a stable propagation is possible under the condition $\eta>1$;

$4)$ at focusing nonlinearity $\left(\beta_{2}<0\right)$ and focusing waveguide $\left(\varepsilon_{1,2}=-1\right)$ a stable propagation of solitons is always possible.

Fig. 1 shows a numerical solution of the system (1) - (2) close to the two-component soliton that is formed at the focusing nonlinearity and the focusing waveguide. The following parameters were used $\beta_{2}=-0.01, \varepsilon_{1,2}=-1, \delta=0, \alpha_{1,2}=0, \gamma_{1}=1, \gamma_{2}=0.5, q_{1}=q_{2}=1$, $\frac{c}{2 n_{1} \omega}=0.1, \frac{c}{4 n_{2} \omega}=0.05$. The incident pulse was taken in the form $\psi_{1}=\mathrm{E}_{1} \operatorname{sech}^{2}(x) \operatorname{sech}^{2}(\tau)$, $E_{1}=10, \psi_{2}=0$. The second harmonic was generated in the process of radiation propagation in the waveguide. This resulted in an optical bullet spread on almost invariably.
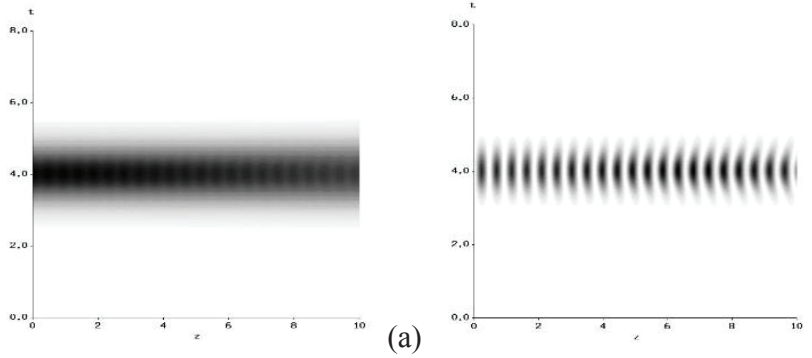

(b)

Fig. 1. The intensity distribution in the cross sections tz demonstrates the formation and propagation of two-component soliton in a focusing waveguide with focusing nonlinearity. (a) fundamental harmonic, (b) second harmonic.

This work is supported by the Russian Science Foundation (Project No 17-11-01157).

\section{References}

[1] A.A. Kanashov and A.M. Rubenchik, Physica D 4, 122 (1981)

[2] B.A. Malomed, P. Drummond, H. He et al., Phys. Rev. E 56, 4725 (1997)

[3] S.V. Sazonov, Phys. of Wave Phenomena 24, 31 (2016)

[4] S.V. Sazonov, J. Phys. Soc. Japan 85, 124404 (2016)

[5] H. Guo, B. Zhou, M. Steinert et al., Opt. Lett. 40, 629 (2015) 